\title{
HOW DOMAIN-SPECIFIC IS MERGE?
}

\author{
DANIEL COOK ${ }^{* 1}$, MAURICIO J.D. MARTINS ${ }^{1,2}$, ARNO VILLRINGER ${ }^{1,2}$ \\ ${ }^{*}$ Corresponding Author: Dan Cook, cookdj0128@gmail.com \\ ${ }^{1}$ Berlin School of Mind and Brain, Humboldt Universität zu Berlin, Berlin, Germany \\ ${ }^{2}$ Neurology Department, Max Planck Institute for Human Cognitive and Brain \\ Sciences, Leipzig, Germany
}

\section{Introduction}

The Minimalist Program (MP) claims that Merge is the core cognitive computation responsible for the recursive hierarchies found in human language and thought (Chomsky, 2017a; Chomsky, 2017b; Hornstein, 2017). Additionally, it claims that Merge is unique to the Language Faculty (Berwick \& Chomsky, 2016; Fitch et al., 2005; Hauser et al., 2002; Hornstein \& Pietroski, 2009; Poeppel, 2017).

Recently, this language-uniqueness view was tested (Martins et al., 2015a) by asking participants to complete a recursive rule-based visual categorization task while performing a phonological working memory task. The verbal task did not interfere with the visual, suggesting that the representation of recursion in vision is not dependent on language domain-specific resources. This study can be criticized, however, for using a verbal task that is not linguistically domainspecific for the MP. More specifically, MP researchers have recently claimed that Merge consists of a linguistically domain-specific cognitive process of (1) lexicalization and (2) concatenation (Zaccarella \& Friederici, 2016; Hornstein, 2017; Hornstein \& Pietroski, 2009). Therefore, in the current study, we operationalize this critique and test the hypothesis that classifying images as wellformed continuations of a recursive rule relies on Merge as defined by the MP. To do so, we used the Visual Recursion Task in a dual-task paradigm that included interference conditions tapping specifically into components (1) and (2) of Merge.

In the Visual Recursion Task (VRT) (Martins, 2015b), participants are shown three successive iterations of a fractal. After the three images appear, two images are shown simultaneously and participants must select the image that best 
corresponds to the correct continuation of the rule established by the first three images. In our study, 48 participants completed the VRT task with and without dual-task interference. The secondary tasks were: 1) A Semantic Fluency condition ('semantic') - tapping into lexical representations - in which participants received a basic category word (e.g. "animals") at the start of a VRT trial and named examples of this category for the duration of the trial; and 2) an Arithmetic Recursion condition ('math') - tapping into recursive concatenation in which participants received a base value (N) at the start of a VRT trial and counted down backwards by three $\left(\mathrm{N}_{\mathrm{i}}=\mathrm{N}_{\mathrm{i}-1}-3\right)$ for the duration of the trial. To compare any specific effects of dual-task interference on the VRT performance, in a second experiment, a new group of participants $(\mathrm{N}=48)$ completed a control task, he Embedded Iteration Task (EIT), a non-recursive iterative rule-based visual task (Martins, 2015b), with the same procedure and dual-task interference conditions as Experiment 1.

\section{Analysis \& Results}

We analyzed accuracy and response times using a Generalized Linear Mixed Model in R (R Core Team, 2013; Bates \& Sarkar, 2006). Subject ID was specified as a random factor to control for repeat measures across fixed factors. We performed model selection by adding factors in a stepwise procedure and comparing log likelihoods. In our final model, VRT trials in the 'math' and 'semantic' dual task conditions were associated with significantly higher error rates $(\mathrm{p}<.05 ; \mathrm{p}<.001)$ and shorter response times $(\mathrm{p}<.05 ; \mathrm{p}<.001)$ compared to trials without dual-task conditions.

Similar to Experiment 1, we analyzed accuracy and response times in the EIT experiment using a Generalized Linear Mixed Model in R. Compared to the EIT task without interference, the 'math' and 'semantic' dual task conditions were associated with significantly higher errors rates $(\mathrm{p}<.001 ; \mathrm{p}<.001)$ and longer response times $(\mathrm{p}<.001 ; \mathrm{p}<.001)$.

Lastly, we combined the VRT and EIT data to investigate the differential effects of the dual-task interference conditions on tasks. The effect of 'math' and 'semantic' interference in the VRT was associated with significantly lower error rates and shorter response times compared to the same categories of effects in the EIT $(\mathrm{p}<.001 ; \mathrm{p}<.001)$.

\section{Conclusion}

Our results suggest that the ability to categorize visual recursion is dependent on some of the abilities enabling Semantic Fluency and Arithmetic Recursion but 
less so than the ability to interpolate fixed positions within a visual sequence (as required by the EIT). This suggests that subcomponents of Merge may be used in the generation of well-formed visual structures following rules. However, the rules may not only be used specifically by recursive operations thought to generate an unlimited amount of novel hierarchies of unbounded depth.

\section{References}

Bates, D. and Sarkar, D. (2006), lme4: Linear Mixed-Effects Models Using S4 Classes, URL http://CRAN.R-project.org, R package version 0.99875-8.

Berwick, R. C., \& Chomsky, N. (2016). Why only us: Language and evolution. MIT press.

Chomsky, N. (2017a). The language capacity: architecture and evolution. Psychonomic Bulletin \& Review, 24(1), 200-203.

Chomsky, N. (2017b). Language architecture and its import for evolution. Neuro. Biobehavioral Reviews. http://10.1016/j.neubiorev.2017.01.053

Fitch, T., Hauser, M. \& Chomsky, N. (2005). The evolution of the language faculty: clarifications and implications. Cognition. 97(2), 179-210.

Hauser, M. D., Chomsky, N., \& Fitch, W. T. (2002). The faculty of language: what is it, who has it, and how did it evolve? Science, 298(5598), 1569-1579.

Hornstein, N. (2017). The Minimalist Program After 25 Years. Annual Review of Linguistics, (0). https://doi.org/10.1146/annurev-linguistics-011817045452

Hornstein, N., \& Pietroski, P. (2009). Basic operations: Minimal syntaxsemantics. Catalan Journal of Linguistics, 8, 113-139.

Martins, M. D. J. D., Muršič, Z., Oh, J., \& Fitch, W. T. (2015a). Representing visual recursion does not require verbal or motor resources. Cognitive Psychology, 77, 20-41.

Martins, M. D., Martins, I. P., \& Fitch, W. T. (2015b). A novel approach to investigate recursion and iteration in visual hierarchical processing. Behavior Research Methods, 48(4), 1421-1442.

Poeppel, D. (2017). The Influence of Chomsky on the Neuroscience of Language. In J. McGilvray (Ed.), The Cambridge Companion to Chomsky (pp. 153-154). Cambridge: Cambridge University Press. doi:10.1017/9781316716694.008

R Core Team (2013). R: A language and environment for statistical computing. R Foundation for Statistical Computing, Vienna, Austria. URL http://www.R-project.org/.

Zaccarella, E., \& Friederici, A. D. (2016). The neurobiological nature of syntactic hierarchies. Neuro. \& Biobehav. Rev.. doi:10.1016/j.neubiorev.2016.07.038 